\title{
Fundamental theories and basic principles of triboelectric effect: A review
}

\author{
Shuaihang PAN ${ }^{1,2}$, Zhinan ZHANG ${ }^{1, *}$ \\ ${ }^{1}$ School of Mechanical Engineering, Shanghai Jiao Tong University, Shanghai 200240, China \\ ${ }^{2}$ School of Mechanical \& Aerospace Engineering, University of California Los Angeles, Los Angeles 90095, USA \\ Received: 18 January 2018 / Revised: 14 March 2018 / Accepted: 10 April 2018 \\ (C) The author(s) 2018. This article is published with open access at Springerlink.com
}

\begin{abstract}
Long-term observation of the triboelectric effect has not only proved the feasibility of many novel and useful tribo-devices (e.g., triboelectric nanogenerators), but also constantly motivated the exploration of its mysterious nature. In the pursuit of a comprehensive understanding of how the triboelectric process works, a more accurate description of the triboelectric effect and its related parameters and factors is urgently required. This review critically goes through the fundamental theories and basic principles governing the triboelectric process. By investigating the difference between each charging media, the electron, ion, and material transfer is discussed and the theoretical deduction in the past decades is provided. With the information from the triboelectric series, interesting phenomena including cyclic triboelectric sequence and asymmetric triboelectrification are precisely analyzed. Then, the interaction between the tribo-system and its operational environment is analyzed, and a fundamental description of its effects on the triboelectric process and results is summarized. In brief, this review is expected to provide a strong understanding of the triboelectric effect in a more rigorous mathematical and physical sense.
\end{abstract}

Keywords: triboelectric effect; triboelectrification; triboelectric nanogenerators (TENGs); interface

\section{Introduction}

The triboelectric effect was the earliest focus in the tribology study. For over 2000 years, its generality and interesting phenomena have attracted significant research interests. In summary, the triboelectric effect is interesting but complex mainly due to the following reasons: (1) Even for a highly charged surface (namely $1 \mathrm{mC} \cdot \mathrm{m}^{-2}$ ), the charge density can only achieve 1 electron unit per $\sim 10^{5}$ surface atoms [1]. (2) Basic questions including which surface will be charged positively and which will have negative charges when two surfaces are brought into contact are still difficult to answer with certainty, and exceptions (i.e., cyclic triboelectric series) from different theories seem exclusive to each other [2]. (3) Multiple factors can work on the same triboelectric process, which adds to the complete analyses by decoupling each dominant parameter for modeling the triboelectric effect. Considering these esoteric aspects in the triboelectric effect, many experiments have been conducted. Though all these results are appreciable, their significance suffers greatly, owing to the lack of more solid theoretical deductions to interpret their phenomena in the triboelectric process [3].

Ironically, with so many tasks to solve and questions to answer, triboelectric devices and applications [4-8] see a far-reaching success in recent years. For example, some previous studies (e.g., into volcanic dusts) have revealed a close relation between environmental phenomena and triboelectric effect $[9,10]$; manufacturing and processing also face the influence of triboelectrication [11]. Indeed, these achievements eagerly need more understanding in theories and

* Corresponding author: Zhinan ZHANG, E-mail: zhinanz@sjtu.edu.cn 
mechanisms in order for a better optimization, a more efficient design, and a greater development potential. Nowadays, newly developed low-band-gap polymers and hard X-ray generators also urgently require a critical review into the triboelectric effect for some novel insights into the applications of these tribo-devices $[12,13]$. Though many simulation and modeling methods are being used to help explain the phenomena, the insufficient physical laws and mathematical description add to the difficulty [14, 15].

This review aims at providing and summarizing the deterministic theories, principles, and mechanisms for the triboelectric effect. Due to the intrinsic characteristics of the triboelectric effect for being system-related and environment-dependent, the review first analyzes the charging media in the tribo-system and summarizes the potential mechanisms for these charging media to transfer (the charging process) in a more mathematical and physical sense (with some novel notions). With this understanding, a more complete list of the triboelectric series is summarized and the abnormal triboelectric sequence phenomena are discussed with clearer mathematical and physical explanations. After all the review into the tribosystem and its related depictions for the triboelectric effect, the important factors influencing the triboelectric performance are systematically studied in the same analytical manner. In addition, we describe the potential applications for these theories and principles for a more comprehensive integration with the triboelectric research and related fields, and how it can be used for a rational design of the tribo-system is also included in each section.

\section{Charging media and charging process}

The triboelectric effect is observable through the measurement of different charging media. An accurate measurement and assessment of the triboelectric effect requires suitable models such as charge injection depth and the evaluation of important parameters such as surface charge density. Given the possible electrical objects, the charging media can be divided into electron, ion, and (nano-)materials. The underlying reasoning for the classification has never been explained systematically, and is summarized here, as shown in Table 1. From our viewpoint in this review, the most obvious difference for these three media is the unit charge carrying capacity. These are the general criteria to decide which media contribute dominantly to the observed phenomena in the triboelectric effect.

\subsection{General important parameters}

\subsubsection{Charge injection depth}

The triboelectric effect will introduce the charge to transfer dynamically, be stabilized dynamically, and be held at the tribological interface. Should the charge be held, the triboelectric interface must have the capacitance characteristics [8, 16, 17]. For example, it has been proved that part of the initial cause of tribological electrification depends on the presence of the internal electric fields in solid insulators [18]. Irrespective of the types of triboelectric contact modes used, the charge will be effective to a certain depth to form the capacitance. This depth is called the charge injection/penetration depth $\lambda$ [19].

For simplicity, the capacitance characteristics in the triboelectric effect can be depicted with $\lambda$ as $[4,16]$

$$
C=\varepsilon S / 4 \pi k \lambda
$$

where $C$ is the equivalent capacitance, $\varepsilon$ is the material's dielectric constant, and $k$ is the electrostatic constant. The simplest configuration with the capacitance in the triboelectric interface is shown in Fig. 1. This material-determined capacitance formed by the

Table 1 The general difference among electron, ion, and (nano-)material.

\begin{tabular}{cccc}
\hline Tribocharging media & Basic unit & Mass & Unit charge carrying capacity \\
\hline Electron & Electron & Low $\left(\sim 10^{-31} \mathrm{~kg}\right)$ & 1 (Medium; Constant) \\
Ion & Ion & $\begin{array}{c}\text { Medium }\left(\sim 10^{-20}-10^{-27} \mathrm{~kg}\right) \\
* \text { No consideration for } \\
\text { macromolecules } / \text { ions }\end{array}$ & $\begin{array}{c}\text { Usually } 1-10 \\
\text { (Large; Ions may carry multiple charges) } \\
\text { (Nano-)material }\end{array}$ \\
\hline
\end{tabular}




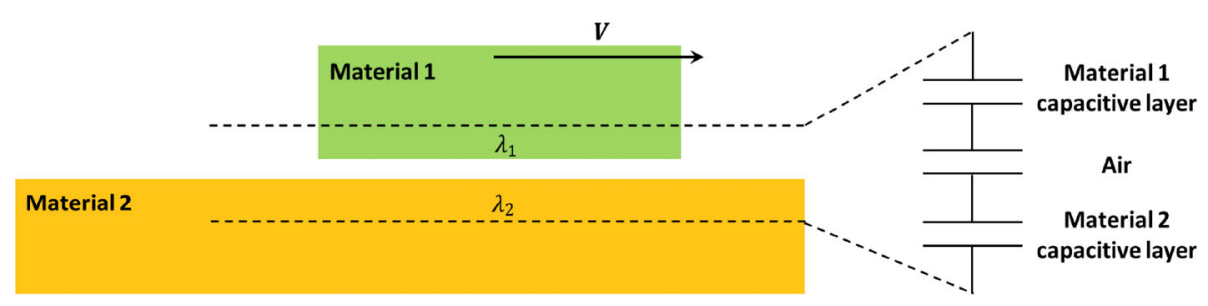

Fig. 1 The capacitive configuration for triboelectric interface.

charge injection depth will possibly interact with interfacial air capacitance, which will influence the dynamical responses of the triboelectric effect (as air has breakdown voltage strength, as stated by Paschen's law) [20, 21].

Note that this configuration is important in understanding many more complicated triboelectric systems including triboelectric nanogenerators (TENGs). By manipulating the triboelectrification and electric induction, there exist four working modes for TENGs, namely vertical contact mode, lateral sliding mode, single electrode mode, and free-standing triboelectric layer mode [16, 22]. These configurations are shown in Fig. 2. Clearly, the capacitive configuration is significant in these devices [23].

If the charge injection depth is larger than the material geometry (i.e., in particle triboelectric systems), the simplified Eq. (1) may not hold, because the charge distribution will be modified to adapt to the limited surface area. For small particles with identical diameters, the capacitance between them

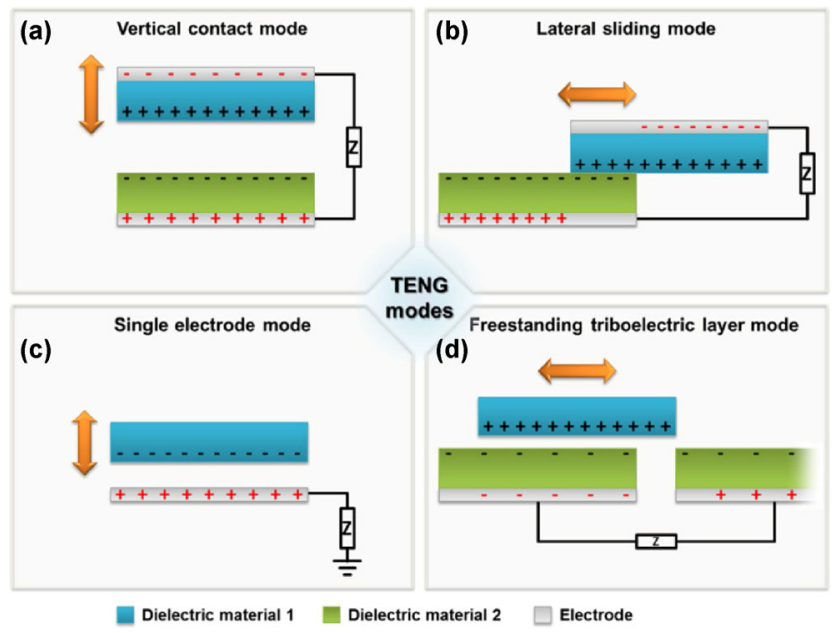

Fig. 2 The four fundamental modes of TENGs: (a) vertical contact mode, (b) lateral sliding mode, (c) single electrode mode, and (d) free-standing triboelectric layer mode [5, 22]. can be calculated as [24]

$$
C=4 \pi \varepsilon_{0} r(\gamma-0.5 \ln (L / r))
$$

where $\gamma$ is the Euler constant and $L$ the distance between two identical particles of radius $r$ [24]. Clearly, the description of particle capacitance is very different from that of area-unlimited surfaces.

\subsubsection{Surface charge density}

Surface charge density $\sigma$ is a parameter for measuring the final charging effects on both surfaces, and it will also significantly determine the triboelectric devices' efficiency. It serves as the basis for analyzing parameters such as the current area power density and volume energy density [25]. Besides, it is a useful parameter to link and analyze surface capacitance characteristics and interface electrical performance, as shown in Eq. (3) [26]:

$$
V=f(Q) \cong f\left(A_{\text {eff. }} \cdot \sigma\right)
$$

where $Q$ is the charge on the surface with an effective area of $A_{\text {eff. }}$. The accumulating charge will then introduce an obvious voltage $V$.

Many previous studies have contributed to accurately measuring surface charge density in triboelectric processes, and some of the data are presented in Table $2[27,28]$.

One critical point is that the final surface charge density can significantly differ from the triggered charge led by the driving force of tribo-contacts. This is mainly because there may exist charge backflow when the contact surfaces are dynamically moving farther or closer, and this is a natural process if the capacitive configuration shown in Fig. 1 is considered [29]. Second, the driving force of the tribo-contacts would not completely contribute to the final surface charge density, as triboluminescence may play a role 
Table 2 Surface charge density after triboelectric charge transfer.

\begin{tabular}{cc}
\hline Material pair & Charge density $\left(\mathrm{C} / \mathrm{m}^{2}\right)$ \\
\hline Chromium-Chromium & $2.02 \times 10^{-8}$ \\
Chromium-Steel & $3.37 \times 10^{-8}$ \\
Chromium-Gold & $6.73 \times 10^{-8}$ \\
Metal-SiO 2 (quartz) & $\sim 10^{-5}$ \\
Metal-NaCl & $5.0 \times 10^{-4}$ \\
Metal-Nylon & $\sim 10^{-3}$ \\
Metal-PTFE & $\sim 10^{-4}-10^{-3}$ \\
Metal-Polyimide & $3.0 \times 10^{-3}$ \\
\hline
\end{tabular}

and consume some of the energy [6, 12].

\subsection{Electron}

When electrons participate as the charging media in the triboelectric process, the work function $W_{\text {surface }}$ is an important factor because it describes the minimum thermodynamic work (i.e., energy) needed to remove one electron from solid to a point in the vacuum, as shown in Eq. (4) [30, 31].

$$
W_{\text {surface }}=-e \cdot \Phi-\varepsilon_{\mathrm{F}}
$$

where $\varepsilon_{\mathrm{F}}$ indicates the Fermi level of electrons in solids and $\Phi$ indicates the electrostatic potential in the vacuum close to the surface ( $-e$ shows negative charge of the electron). Nowadays, work functions can be measured with photoemission spectroscopy and the Kevin-Zisman method [32, 33].

Because fermi level will be introduced for the work function calculation, metals will be considered as important surface materials if the electron transfer dominates [31,34]. Note that an (equivalent) work function can be meaningful for insulators including polymers such as polytetrafluoroethylene (PTFE) and polycarbonate when they serve as a tribosurface $[31,35]$ (though some studies have considered the electron transfer as trivial for the charging process, due to the mismatch of the correlation between the insulator ionization potentials and the charging behavior [36]).

During the process, the driving force of the electron transfer in the triboelectric effect is proven to be a tribologically introduced phonon-electron interaction [30]. The primitive relation of coupling energy between the tribological heat and the transferred electron is expressed as

$$
\Delta S \propto \Delta \omega \cong \omega_{0} \cdot \bar{a} \cdot \Delta k=\frac{4 \pi \omega_{0} S}{N \bar{a}} \cdot \delta a \propto \delta a \propto f\left(F_{\mathrm{f}}, F_{\text {atom }}, \varepsilon, T \ldots\right)
$$

where $\Delta S$ is the entropy change/energy state change introduced by the friction contact and can be a good indicator to correlate with the surface density of states, $D_{s} ; \omega_{0}$ is the lattice natural well-defined frequency, which demonstrates the phonon energy levels; $\bar{a}$ is the average lattice parameter; and $\Delta k$ is change in the reciprocal lattice parameter corresponding to the lattice momentum. $f\left(F_{\mathrm{f}}, F_{\text {atom }}, \varepsilon, T \ldots\right)$ denotes the influence from external factors including strain $\varepsilon$ and temperature $T$.

According to the experiments conducted with metal-insulator tribo-pairs, the surface density charge $\sigma$ can be correlated with the work function using Poisson equation, as follows:

$$
\sigma=\alpha \cdot \varepsilon \cdot\left(\frac{\Phi_{\mathrm{M}}-\Phi_{\mathrm{I}}}{\lambda}\right)
$$

where $\alpha$ is the leading factor, whose value in metaldielectric cases is $1.77 \times 10^{-13} ; \varepsilon$ is the dielectric constant for insultors; and $\Phi_{\mathrm{M}}$ and $\Phi_{\mathrm{I}}$ demonstrate the work function for metal and insulator, respectively. The Poisson equation is dynamically verified from the fact that the triboelectric effect is a process in which charging and discharging are continuously coupled, and this relation is widely used in the particle triboelectric process simulation and analyses [2,37].

Following the thoughts of energy levels (including work function), for metal-dielectric-metal tribo-pairs (e.g., alkanethiol monolayer-coated $\mathrm{Au}(111$ on $\mathrm{Au}$ ) [38]), the main role of friction in electron transfer is to generate electron-hole pairs in mid-gap states near the Fermi surface (which explains why the work function is important) in normally insulating materials if they are in monolayer shape [38,39]. Given the dielectric electronic parameters, the tribo-current (i.e., the conductance of tunneling electrons transferred) can be calculated as follows [38]:

$$
G_{f}=k_{t} \cdot \exp \left(-1.025 \cdot \sqrt{\frac{E_{g}}{2}} \cdot z\right)
$$


where $G_{f}$ denotes the conductivity value in Siemans (S) and $k_{t}=\frac{2 e^{2}}{h}$ is the quantum conductance. $E_{\mathrm{g}}$ (e.g., $\sim 9 \mathrm{eV}$ for alkanethiol) and $z$ are the material parameters, namely the band gap of the dielectric film and the dielectric film thickness, respectively. Here, it is assumed that the fermi surface is located in the middle of HOMO and LUMO [30, 38].

Even in particle triboelectrification (e.g., in pharmaceutical manufacturing), electron transfer is important. According to the nonequilibrium analysis conducted by Kron, Cubero, and Lacks et al. [40-42] an excess of electrons trapped in the more easily transferred (presumably higher-energy) state is the driving force for the particle triboelectric initiation, as shown in Fig. 3 [1, 42]. This conclusion is drawn with the assumptions and confirmations from other experiments and studies, which all verify that the density of the available energy states is high enough and not limited in even particle media, and that the charge transferred would not be limited by it $[19,43,44]$.

\subsection{Ion}

Because electron transfer and ion transfer have several similarities, importance is cast onto how to distinguish the electron or ion source for charging in the triboelectric process. Even though the effective work function can be used to explain the metal-insulator tribo-pairs' electrification, in case of insulator-insulator tribo-pairs, electron transfer is often believed to be impossible [2].

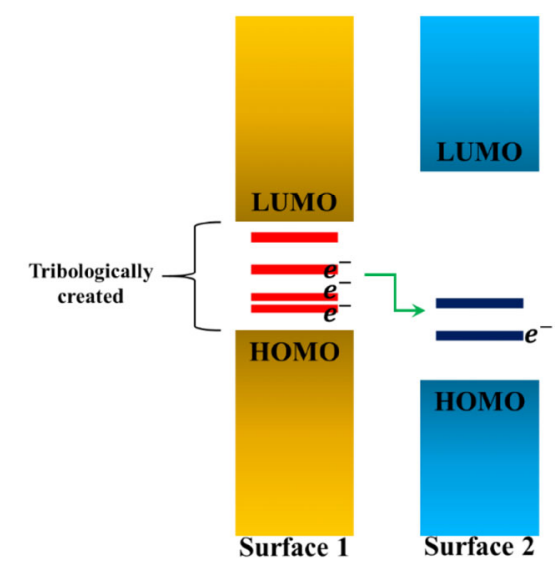

Fig. 3 Tribologically induced non-equilibrium states to accommodate and aid charge transferring in two surfaces (mainly insulating surfaces).
As stated above, how to distinguish electron and/or ion transfer is important. As is known, the redox reactions are the signature for electron transfer because it leads to the valence change. Therefore, many experiments depend on the redox reactions to indicate the dominant charge transfer process $[45,46]$. In turn, dependent on these experiments, the electro-potential of the surface (compared to $\mathrm{H}+/ \mathrm{H}_{2}(0 \mathrm{~V}$ versus $\mathrm{NHE})$, which is not necessarily equal to the electro-potential energy) can be more quantitatively determined [46].

Note that the ion transfer in the tribo-charging process dominates the insulator charging surface. In the ion transfer process, because the surface may have strongly bounded ions of one charge polarity and loosely bounded ions of the other polarity. When the tribological contact is initiated, the imbalance affinity with various ions will lead to the transfer of certain types of ions, and as a result, accumulate charge on the surface [47]. This relation, with analyses into entropy and electrostatics, is depicted in Eq. (8) $[2,48]$.

$$
\frac{n}{N-n}=\exp \left(-\frac{n d e^{2}}{\varepsilon_{0} k_{\mathrm{B}} T}\right)
$$

where $N$ is the ionic functional groups/units per area and $n$ is the anion number density. $d$ denotes the distance between the two "planar, equally and oppositely charged" surfaces [49]. Note that the term $\frac{n d e^{2}}{\varepsilon_{0}}$ indicates the electrostatic free energy in ion transfer, which serves as the determining factor for either positive or negative ion transfer.

Specifically, if the ions transfer at the metal-polymer interfaces dominantly, the metal surface property is relatively trivial and the charge is believed to mainly stem from the protons dissociated from the ambient water residing on the contact surface. The fitting relation between the contact charge (density) $q$ and the equilibrium constant for the association of a proton $\left(\mathrm{pK}_{\mathrm{b}}\right)$ of insulators (especially polymers) is [36]

$$
\log (q+1) \cong 0.0305 \cdot \mathrm{pK}_{\mathrm{b}}+0.2731 \text { (with } R^{2}=0.91 \text { ) }
$$

where $R^{2}$ demonstrates the quality of fit.

Moreover, in metal-polymer cases, ion transfer can 
also be considered from the metal (mainly as donors) side. The donor number $(\mathrm{DN})$ values reflect the enthalpy associated with the coordination reaction between the metal cations and repeat unit of polymers [50]. Then, the fitting relation between the charge density $q$ and DN values is [36]

$$
\log (q)=0.09 \cdot \mathrm{DN}-0.36\left(\text { with } R^{2}=0.80\right)
$$

Equations (9) and (10) follow a similar trend for describing the ion transfer in metal-polymer cases, and it is a natural analogy, because $\mathrm{pK}_{\mathrm{b}}$ and $\mathrm{DN}$ parameters are related to the ability of the chemical site to donate a pair of electrons to either a proton center or a metal cation center, respectively, as illustrated in Fig. 4.

\section{4 (Nano-) material}

Material transfer to cause charging is believed to occur when vigorous rubbing or pressing is introduced [29, 51]. For example, when some polymers are rubbed with friction, an exchange of deeper layer materials occurs [52]. (This helps explain why even the same polymers can be charged by tribo-contacts; it is because even identical polymers will have different material compositions at each depth, which account for the same material charge transfer [29].)

Moreover, material transfer is possible if dynamical changes in the surfaces and tribological environments exist, because materials will tend toward a lower potential energy state and these dynamical changes can lead to the potential energy minimum shifts [53, 54]. For example, one dynamical change (in quasistatic form) can be strain and its corresponding potential energy form (near the potential energy local maximum, i.e., the local energy barrier at interface) is

$$
E_{\mathrm{p}}=f(\varepsilon)=\delta^{2} \cdot \varepsilon-\frac{1}{3} \varepsilon^{3}
$$

where $\varepsilon$ is the actual strain and $\delta$ is the strainrelated parameter (which has a relation $\delta=f_{1}(\varepsilon)$ ). When $\delta$ evolves with the increased strain and changes

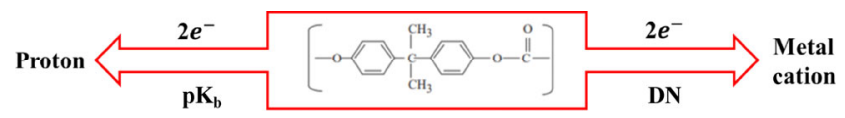

Fig. $4 \mathrm{pK}_{\mathrm{b}}$ and $\mathrm{DN}$ parameters in ion transfer relationships. to be below 0 , the local potential energy barrier will be demolished and the materials (originally in the nearest locations of minimum potential energy and hindered by this energy barrier) will begin to migrate to the new energy-stable developed by this dynamical tribological environment change, as shown in Fig. 5 [53].

This understanding from catastrophe theories can be extended to many other factors, including temperature, density, and normal loads, for a more complete discussion of (nano-)materials' transfer in the triboelectric process. This is because the charging process initiated by material transfer can be viewed as a balance or nonequilibrium between the driving force of friction and the energy barrier erected in accordance with various physical properties.

After the material transfer prerequisite from energy consideration is fulfilled, more recently, studies have been conducted to investigate the material removal rate both physically and chemically $[55,56]$. The material wear rate is physically deduced and the load stress is calculated to dominate in the wear process, as follows $[55,57]$ :

$$
\begin{gathered}
\Gamma=\Gamma_{0} \cdot \exp \left(-\frac{\Delta G_{\text {act }}}{K_{\mathrm{B}} T}\right)=\left\{\Gamma_{0} \cdot \exp \left(-\frac{\Delta U_{\text {act }}}{K_{\mathrm{B}} T}\right)\right\} . \\
\exp \left(\frac{\sigma_{\mathrm{N}} \cdot \Delta V_{\text {act }}}{K_{\mathrm{B}} T}\right)
\end{gathered}
$$

where $\Gamma$ (in the unit of a.u./s) is the actual wear rate considering both chemical wear nature (shown by the term $\left.\exp \left(-\frac{\Delta U_{\text {act }}}{K_{\mathrm{B}} T}\right)\right)$ and physical load stress (shown by the term $\exp \left(\frac{\sigma_{N} \cdot \Delta V_{\text {act }}}{K_{\mathrm{B}} T}\right)$ ), and $\Gamma_{0}$ is the

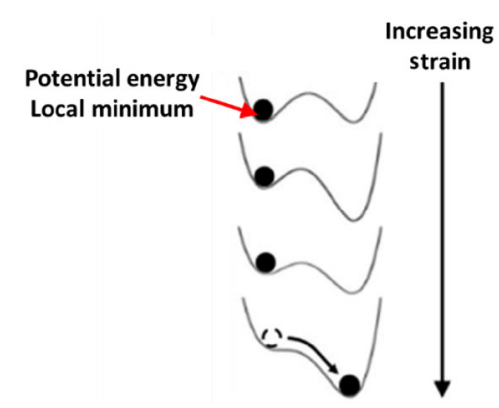

Fig. 5 Schematic of changes in the potential energy landscape with strain. 
pre-factor as an effective attempt frequency. $\Delta G_{\text {act }}$ is the total free energy of activation for wear, which is equal to $\Delta U_{\text {act }}-\sigma_{\mathrm{N}} \cdot \Delta V_{\text {act }}$. In this sense, $\Delta U_{\text {act }}$ indicates the internal free energy of activation by chemical processes and $\sigma_{\mathrm{N}} \cdot \Delta V_{\text {act }}$ links the normal load stress $\sigma_{\mathrm{N}}$ (governed by Eq. (13)) and the effective activation volume $\Delta V_{\text {act }}$ to provide the tribological contribution to the material removal process [55].

$$
\sigma_{\mathrm{N}}=\frac{F_{\text {applied }}+F_{\text {adhesive }}}{A_{\text {contact }}}
$$

Through the above relations, when a (nano-)material is analyzed for the triboelectric effect, we can more confidently expect exponentially coherent relations among the load stress, material transfer (mainly the wear rate), and tribo-charging value [34, 38].

Finally, as listed in Table 1, tribo-charging through both ion and (nano-)material has the varying unit charge carrying capacity, which also complicates the discriminations between ion and (nano-)material transfer. To achieve the goal, some methods have been proposed, one of which is described as follows: The tribo-charging characteristics of polymers are insensitive to the carbon black incorporation, whereas metals become much triboelectrically susceptible to carbon black composition. Therefore, carbon black can be added and traced, and the change in triboelectric signals caused by carbon black can be the judgment. Therefore, the use of carbon black can help distinguish whether the material transfer will contribute to the tribo-charging process [51].

\section{Triboelectric series}

The most important notion in the triboelectric effect is to empirically set up the triboelectric series, which gives a general idea of how the charging will take place when any two materials are brought into contact through rubbing, pressing, or friction. This helps simplify the triboelectrification analyses for other applications including TENGs (e.g., which use $\mathrm{Al}$ and PTFE as the potential surface materials [58]). A more detailed and complete triboelectric series list is provided below in Table $3[1,27]$. A more scientific segregation for the triboelectric series based on pure
Table 3 Triboelectric series short summary.

\begin{tabular}{cc}
\hline More positively charged $(+)$ & $\Delta$ \\
\hline Rabbit's Fur, Hair & Brass \\
Glass & Silver \\
Mica & Gold \\
Wool & Polyester (PET) \\
Nylon & Polystyrene \\
Lead & Acrylic \\
Silk & Polyvinyl chloride \\
Aluminum & Polyvinyl chloride w/plasticizer \\
Paper & Polyethylene \\
Wood & Polypropylene \\
Amber & Polytrifluorochloroethylene \\
Sealing wax & Teflon (PTFE) \\
Rubber Balloon & Silicon Rubber \\
Nickel & Ebonite \\
Copper & More Negatively Charged (-) \\
$\nabla$ & P
\end{tabular}

* Pure polymers (not mixtures) are marked in red. Pure metals as the segregation points are marked in blue.

metals as the division points can be referred to in Ref. [49].

The intensive research has cast light on some triumphing trend explanation for the above triboelectric series. It has been confirmed that polymers having strong tendencies to charge positively have high dielectric constants, and are highly polar and hydrophilic [29]. For example, it is found that polymers with oxygen functional groups (more polar) will be charged more positively than those with nitrogen functional groups [36]. This fits well with the sequence shown in Table 3, supported by Fig. 6 .

However, while triumphing over the fitness between the material selection and the triboelectric series, the research into its sequence is still not enough. Some contradictory experiments are reported; for example, the triboelectric sequence of polyethylene and polypropylene is confusing. Besides, both interesting and esoteric phenomena are observed, two of which with the huge puzzle for scientists are the cyclic triboelectric series and tribo-electrification of identical materials. For these observations, still insufficient evidence is proved to support the theories and no consistent experiment results are convincing. 


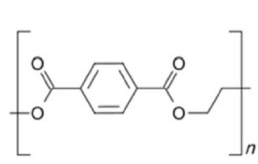

Polyester

(PET)

4 oxygen in unit;

Totally polar unit

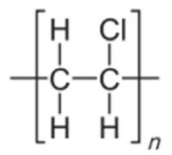

Polyvinyl

chloride

Polar by $-\mathrm{Cl}$

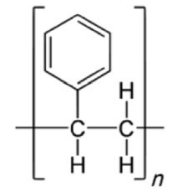

Polystyrene

Polar by $-\mathrm{Ph}$

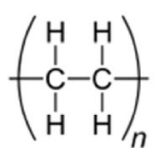

Polyethylene Less polar

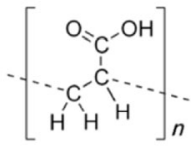

(Poly)acrylic

2 oxygen in unit; Polar by $-\mathrm{COOH}$

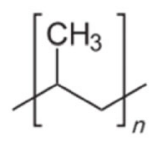

Polypropylene Less polar
Fig. 6 The configuration and characteristics of the polymers.

\subsection{Cyclic triboelectric series}

One representative example of the cyclic triboelectric series is shown in Fig. 7 [2, 49]. One direct observation for the cyclic triboelectric series lies on the co-existence of materials of different types, different structures, and different compositions. As shown, even paper and cotton with the same main composition of cellulose to different ratios will have a different position in this series.

Therefore, only one thing in consensus is that the appearance of the cyclic triboelectric series is a combination of coupling different charge media, charging modes, and the electrification mechanism [2]. The series from silk to glass is easier to understand because they exhibit an obvious increase in the concentration of oxygen in their composition, which

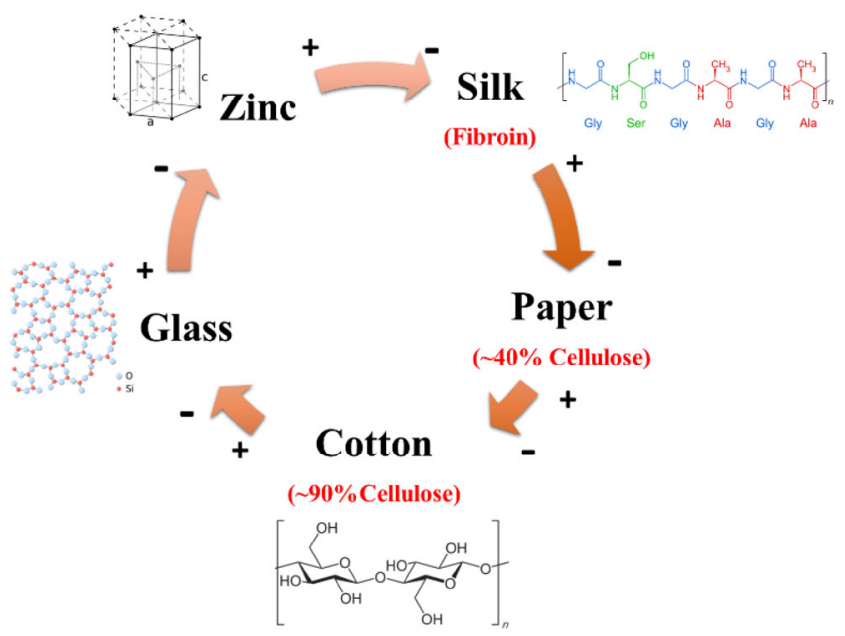

Fig. 7 Demonstration of cyclic triboelectric series. fits the primitive trend illustrated in Section 3. Hence, the difficulty will be reduced to only make the link among "Glass-Zinc-Silk" part to couple and decouple different mechanisms for the cyclic phenomena.

\subsection{Tribo-electrification of identical materials}

If the two surfaces are identical in materials and their general composition, triboelectrification, according to the common senses, should be ignorable, or at least random [59]. However, this is not the case for many experiments, which report the same reproducible triboelectric trend on certain material surfaces. Though identical materials' triboelectrification seemingly breaks the triboelectric series generality, it indicates the impacts of the hidden coupled factors.

An important theory trying to explain the triboelectric effect on identical surfaces is the asymmetric contact theory $[1,60]$. Non-equilibrium surface states (of electrons, ions, etc.) are essential in that they help provide the driving force to transfer the charging media in higher non-equilibrium surface states on one surface to lower states on the other [30]. Therefore, the probability of the charging will be proportional to the surface density difference:

$$
P(\text { surface } 1 \rightarrow \text { surface } 2) \propto \sigma_{1}-\sigma_{2}
$$

where $P$ (surface $1 \rightarrow$ surface 2$)$ denotes the net charge transfer from surface 1 to the surface of the same material, and $\sigma$ is the area density of the net transferring media in higher energy states (Fig. 3).

According to this theory, when two identical surfaces are contacted, the deterministic triboelectrification can occur in the following fashion: The same part of surface 1 contacts the different parts of surface 2 continuously and the charging process will be always in the same direction, with the depletion of the nonequilibrium states of surface 1 acting as the driving force.

$$
\left|\sigma_{1}-\sigma_{2}\right| \rightarrow 0 \text { with } \sigma_{12} \equiv \sigma_{2}(\text { non }- \text { contact })
$$

Then, the surface area will play a significant role when triboelectrification on identical surfaces takes place [1].

Besides, the triboelectric process on identical materials can be understood from a statistical viewpoint (i.e., 
symmetrical electrification, where Eq. (15) is already satisfied). In this case, it can be expected that

$$
\overline{\sigma_{1}} \cong \overline{\sigma_{2}}
$$

Due to the statistical variation expectation theories, the probability of directional triboelectric charging will be variation/deviation-determined [59, 61], in the form of

$$
P(\text { surface } 1 \rightarrow \text { surface } 2) \propto \sqrt{A_{1} \cdot \overline{\sigma_{1}}}-\sqrt{A_{2} \cdot \overline{\sigma_{2}}}
$$

where $A_{1}$ and $A_{2}$ are the areas of surface 1 and surface 2 , respectively, and $\sqrt{A_{i} \cdot \overline{\sigma_{i}}}$ is the expected variation value, in a statistical sense.

In summary, triboelectric series are an efficient tool for triboelectric analyses, which provide a useful picture of how a tribo-pair will contribute to the final charging. The logic scheme for understanding the triboelectric series and its exceptional cases is summarized in Fig. 8.

\section{Important factors}

This section focuses on what factors as controllable variables are influential, or even dominant, in the triboelectric process and how they determine the triboelectric series externally, which can help explain some contradictory results to list the triboelectric series. The scheme of this chapter is summarized in Fig. 9. Generally, the factors can be divided into two categories by the system composition consideration: surface properties and environmental influence.

Surface properties are the characteristics of the tribo-systems themselves, which are determined upon the setup of the tribo-pairs. Hence, materials and their

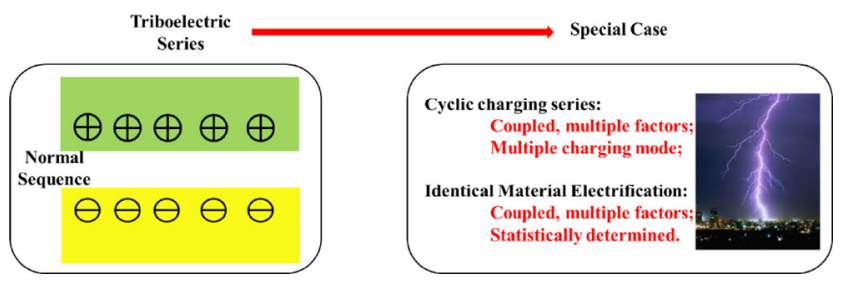

Fig. 8 Understanding for triboelectric series ("Special Case" refers to the triboelectric series composed of (a) identical materials in Section 3.1 and (b) cyclic material loop in Section 3.2).

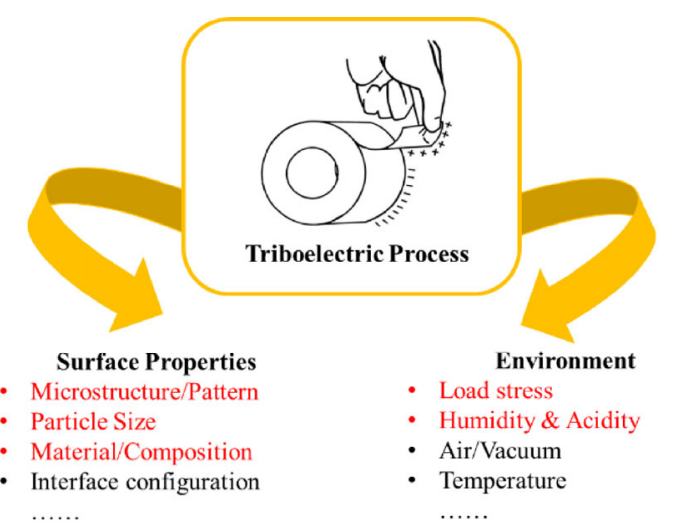

Fig. 9 The schematic illustration of the parameters influencing triboelectric process.

compositions are the leading factors in the discussion on the triboelectric effect. This has been included in Sections 1 and 2, and will not be further detailed in this section. The microstructure and pattern also need immediate attention because they can be influenced by the material composition, and key tribological indicators such as surface roughness can interactively tangle with the changes in microstructures and surface patterns. Meanwhile, as particle contact and triboelectrification is a practical topic in varied industries, particle size will be considered separately as a key factor to determine the triboelectric series in this section.

In case of environment contributions, load stress, humidity, and acidity are non-ignorable. Load stress is fundamental in a way that it links the mechanical and electrical behaviors. Indeed, understanding the mechanical responses can be a strong basis and useful tool for further discussion of the triboelectric performance, as friction and contact are the sources for the charging process [34]. Humidity and acidity are of immense importance, and will not be discussed independently. We conducted the discussion together on purpose, because the main idea of humidity and acidity impacts on the triboelectric effect is the concern with ion/polarity generation and chemical reaction [2]. Therefore, their importance roots in factors such as the possible subsequent surface composition changes.

Other factors are also meaningful for obtaining a complete picture of the triboelectric series. For instance, the charge in the vacuum on the tribo-surface is usually higher than that in the atmosphere [36]. The triboelectric effect is also a temperature-dependent 
process [58]. However, due to the scope of our review, they will not be discussed in equal detail here.

\subsection{Microstructure/pattern/geometry}

As stated in Section 2, an interesting phenomenon in the triboelectric effect is the tribocharging of chemically identical materials as the friction contact surfaces [61]. Different explanations and theories are provided to understand the electrification, among which pattern and geometry are believed to play a vital role. Wang et al. [53] proved that the differences in the microstructure of chemically identical materials trigger distinct tribo-charging behavior. In this sense, as a strained surface will exhibit different microstructures due to voids and seams (which can be scaled from nano- to micrometers) and the different microstructures will trigger different surface potential energy minimum according to catastrophe theories [53,54], as shown in Fig. 5, the strained surface may exhibit different triboelectric behavior [20,53].

Except for the microstructure effects, the surface patterns can be tuned by the surface roughness, which can also tune the triboelectric behavior. Indeed, different area roughness will make the tribo-charging more local and the mosaic pattern for charges (whether positive or negative) will exist [62].

Interestingly, the final charge distribution pattern, irrespective of the micro or macroscale [61-63], will also be affected, and in turn affect the triboelectric charging process. This indicates that the local charge can be much greater in magnitude than the net average charge on the surface, and that the net triboelectric effect represents an average of the possible contributions from both positively and negatively charged local regions [1].

\subsection{Particle size}

For the same materials in particle shape, when the particles are in different sizes, opposite polarities will form to help with the bipolar charging process [42, 64]. The size-dependence of particle triboelectrification is observed in both natural phenomena $[10,65]$ and industrial processes $[11,66]$, which gives it priority in research fields and for which many modeling studies have been conducted $[9,67,68]$.

Detailed studies on particle sizes have suggested that the magnitude of the charge increases continuously with a decrease in particle size, as indicated in Table 4 [69]. A possible reason is thought to be the adhesion of fine particles $(<40 \mu \mathrm{m})$ to coarse particles, which eventually enhance the surface roughness of the coarse particles, similar to the effects of the pattern/ microstructure shown in Table 4.

Except for the pure charge (density) analyses, triboelectric series analyses have also been given equal importance [64, 70,71]. These experiments and simulations confirm the general trend that large particles tend to charge positively, while small particles tend to charge negatively. The theory behind this trend can be summarized from the following several aspects [24, 70].

For a bimodal mixture particle system (as a simplest case) of masses in $m_{\mathrm{L}}$ and $m_{\mathrm{s}}$, respectively, the mass fraction $w_{\mathrm{B}}$ is defined as $\frac{m_{\mathrm{L}}}{m_{\mathrm{L}}+m_{\mathrm{s}}}$. With solid-sphere simplification for particles, the probability $P\left(D_{i}, D_{j}\right)$ of a collision between two particles with sizes $D_{i}$ and $D_{j}$, respectively, will lead to the collision fraction occurring between a large particle and a small particle $f_{\text {LS }}$ and give it the form of [70]

$$
f_{\mathrm{LS}}=\frac{0.5\left(\frac{1+D_{\mathrm{L}} / D_{\mathrm{s}}}{2}\right)^{2} \cdot x_{\mathrm{s}} x_{\mathrm{L}}}{x_{\mathrm{s}}{ }^{2}+\left(D_{\mathrm{L}} / D_{\mathrm{s}}\right)^{2} \cdot x_{\mathrm{L}}{ }^{2}+0.5\left(\frac{1+D_{\mathrm{L}} / D_{\mathrm{s}}}{2}\right)^{2} \cdot x_{\mathrm{s}} x_{\mathrm{L}}}
$$

where $D$ indicates the diameters for either particle (in large or small size) and $x$ indicates the number fractions for either large or small size in a charged state (positive or negative). Then, the charge segregation factor $\xi$ is calculated as

$$
\xi=\sqrt{\frac{x_{\mathrm{L}}^{+}}{x_{\mathrm{L}}^{-}} \cdot \frac{x_{\mathrm{S}}^{-}}{x_{\mathrm{S}}^{+}}}
$$

Table 4 Effect of particle size on tribo-charging.

\begin{tabular}{cc}
\hline Particle size (um) & Charge (nC/g) \\
\hline $335-500$ & 10 \\
$250-355$ & 28 \\
$125-150$ & 50 \\
$90-125$ & 65 \\
\hline
\end{tabular}


where $x_{i}^{\delta}$ depicts the number fraction of particles in size $i(i=\mathrm{L}, \mathrm{S})$ to be charged in $\delta$ state $(\delta=+$ positively, - negatively). Using this model from binary viewpoint, $\xi>1$ is proved to be valid, and the trend that large particles tend to charge positively (e.g., $x_{\mathrm{L}}^{+}$is large and dominates) and small particles tend to charge negatively can then be physically understood and statistically solved.

Meanwhile, the size effect can also show up in how the particle triboelectrification can respond to the environment they are in. For example, under an external electric field, for a spherical dust grain of radius $r$ and a homogenous electric field $E_{0}$ perpendicular to the surface, the induced charge is expressed as

$$
Q=4 \pi \varepsilon_{0} \cdot \alpha E_{0} r^{2}
$$

where $\alpha$ is the electric field-related factor showing the geometry effect from the field and $r$ denotes the particle radius. This model is significant because when particles are electrified, any single particle is placed in a series of linearly addable particle-introduced electric fields [24].

\subsection{Load stress}

It has long been proved that stress can produce ions $[72,73]$ and electrons [74] and greatly affect their behavior [14], because the load stress is where friction can be introduced. For instance, different loads can involve different charging mechanisms: when the frictional contact is gentle and brief, (nano-)material transfer can be precluded [51]. More theoretically, the energy barrier analyses and wear rate correlation from Eqs. (11) and (12) all demonstrate the possibility that the triboelectric effect is highly load-dependent [34].

Though the exact depiction for the triboelectric effect from load stress behavior is incomplete, many applications have already utilized this important factor to compromise or take advantage of the triboelectric process. TENGs designed with a triple cantilever is used to harvest the vibration energy, which is operating under the variable normal load condition [75]. Especially when the electromechanical phenomena are involved (e.g., the coupling between the piezoelectric effect and charge separation), the understanding of the role of the normal load is essential [76-78].
Since more load stress effects are analyzed for a pure mechanical tribological process for now, more notions into the load dependence in the triboelectric process would be beneficial to understanding the intrinsic roles of normal force as the initializing potential.

\subsection{Humidity and acidity}

Humidity and acidity have obvious significance owing to their strong effects on surface electrical conductivity and capacitance configuration (for example, the charge leakage required to achieve a dynamical balance in the triboelectric effect will be affected [1]; in real applications, salts including quaternary ammonium [29] can then be used as the charge control agent) $[79,80]$.

The acid-alkaline theory $[18,27]$ is both a supplementary and an extensive theory to understand the triboelectric effect influenced by the environment. Here, the acid and base are in the scope of Lewis classification [2], and works significantly for insulator tribo-pairs. As stated in Section 1.3, the equilibrium constant for the dissociation of a proton $\left(\mathrm{pK}_{\mathrm{b}}\right)$ of insulators (especially polymers) is found to determine the position in the triboelectric series.

Humidity and acidity are similar because the ion/polarity generation and chemical reactions play a clear role $[2,15]$. For instance, the process linking humidity and acidity can work in the following way: when a hydroscopic surface (or a surface with hydroscopic groups) is placed in the humid environment before/during tribo-contact, more water or salt will be attracted to form a conducting layer (in reality, it might still not be conductive), which may help charge leakage, and thus, influence the charging process [1]. This is illustratively shown in Fig. 10. However, note

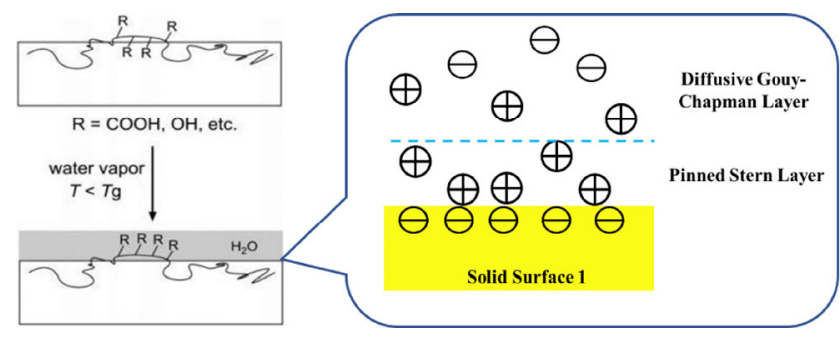

Fig. 10 The schematic illustration about the influence of humidity onto organic insulator surface during triboelectric process [2, 84]. 
that the general trend of how tribo-charging is affected by this humidity- and acidity-introduced layer is not reached consensus yet [81-83].

After humidity and acidity (with their functional groups) are attached to the original tribo-surfaces, the solid-liquid interface will be naturally created $[2,84]$. Why we stress on this newly introduced two-phase interface is because the tribo-characteristics have been changed away from solid-solid contact, and it turns out to have various electrical behaviors in the triboelectrification process.

Some useful theories have been established for the simplest case of water residing on the solid surface, and the surface electrical variation is observed for possible control of the triboelectric process ("singlesurface" analysis) [84]. In this case, the electrical double layer (with the Stern layer and the Gouy-Chapman layer) will be formed. Without loss of generality, the charging distribution is set as shown in Fig. 10. Due to the electrochemical potential balance of each ionic group $\mu$ demonstrated in Eq. (21), the anion and cation concentration $c_{ \pm}$can be calculated by a thermophysical consideration, and the relation is shown in Eq. (22).

$$
\begin{gathered}
\mu_{i}=\mu_{i 0}+R T \ln c_{i}+F q_{i} \varnothing \\
\frac{c_{ \pm}}{c}=\exp \left(\mp \frac{q \varnothing}{k_{\mathrm{B}} T}\right) \text { with } \mathrm{c}=c_{-}-c_{+}
\end{gathered}
$$

where $F$ denotes the Faraday constant in electrostatics, $q$ is the valence charge of each ion, and $\varnothing$ is the (local) electrical potential.

Given the electrical gradient and the local charge density, the Poisson equation can be modified to

$$
\nabla^{2} \varnothing=\frac{2 c q}{\varepsilon \varepsilon_{0}} \cdot \sin h\left(\frac{q \varnothing}{k_{\mathrm{B}} T}\right)
$$

By solving Eq. (23) to obtain the Debye length, we can see a clear disturbance to the original electric field as well as electrical potential distribution vertical to each surface. When more information including the proton dissociation energy, surface ion adsorption energy, and surface free energy is provided [36], the analytical solution to the humidity-acidity effect can be expected.

Based on the above arguments, we can easily link the triboelectric effect with the wetting behaviors of the surfaces, as the wettability of surfaces indicates how and how well these acid-base pairs or groups can attach to tune the surface properties, and thus, change the triboelectric performance [1, 80, 85]. For example, higher oxidation of polystyrene leads to lower contact angle (thus higher wettability) and determines the rate of charging (with the tribo-pairs formed by metals and organics) [81]. Other experiments confirm this relation by treating the surface with UV light to cause the contact angle change and then investigating the charging behavior [86].

\section{Summary}

This is the first review to place great importance on the fundamental theories and basic principles for more comprehensively understanding the triboelectric effect and its interaction with its variable environments. The first section gives a general picture of the electrical property (e.g., capacitive characteristics) of the triboelectric system and reviews the different charging media (electron, ion, and (nano-)material) and their governing rules in detail. With the mathematical and physical descriptions of these charging modes, the triboelectric series are discussed, with a rational focus on the special cases including cyclic triboelectric series and triboelectrification on identical materials. Finally, the triboelectric effect is a combination of both system features and environment influences, which make the discussion on the important factors following the sequence of structure effect, (particle) size effect, load dependence, and humidity dependence more reasonable. In brief, this review casts light on how the fundamental theories are developed and confirms their deterministic functions in designing more effective triboelectric systems with these relatively precise mathematical and physical descriptions.

\section{Acknowledgement}

This work was supported by the National Natural Science Foundation of China (No. 51575340), State Key Laboratory of Solid Lubrication (No. LSL-1604) and the Shanghai Academy of Space TechnologyShanghai Jiao Tong University Joint Research Center 
of Advanced Aerospace Technology (USCAST2016-13). The authors gratefully acknowledge Tianlu Wang (PhD, ETH Zurich), Peng Zhang (PhD, UCLA), and Ning Yu (PhD, UCLA) for their useful comments and proofreading.

Open Access: The articles published in this journal are distributed under the terms of the Creative Commons Attribution 4.0 International License (http:// creativecommons.org/licenses/by/4.0/), which permits unrestricted use, distribution, and reproduction in any medium, provided you give appropriate credit to the original author(s) and the source, provide a link to the Creative Commons license, and indicate if changes were made.

\section{References}

[1] Lacks D J, Sankaran R M. Contact electrification of insulating materials. J Phys Appl Phys 44: 453001 (2011)

[2] McCarty L S, Whitesides G M. Electrostatic Charging due to separation of ions at interfaces: Contact electrification of ionic electrets. Angew Chem.Int Ed 47: 2188-2207 (2008)

[3] Shafeek S, Luo J. Theoretical and numerical analysis of triboelectric nanogenerators for self-powered sensors. In 2016 5th International Conference on Electronic Devices, Systems and Applications (ICEDSA), 2016: 1-4.

[4] Zhu G, Peng B, Chen J, Jing Q, Wang Z L. Triboelectric nanogenerators as a new energy technology: From fundamentals, devices, to applications. Nano Energy 14: 126-138 (2015)

[5] Wang Z L. Triboelectric nanogenerators as new energy technology and self-powered sensors-Principles, problems and perspectives. Faraday Discuss 176: 447-458 (2015)

[6] Camara C G, Escobar J V, Hird J R, Putterman S J. Correlation between nanosecond X-ray flashes and stickslip friction in peeling tape. Nature 455: 1089 (2008)

[7] da Silva E T S G, Santhiago M., de Souza F R, Coltro W K T, Kubota L T. Triboelectric effect as a new strategy for sealing and controlling the flow in paper-based devices. $L a b$ Chip 15: 1651-1655 (2015)

[8] Ye U Y, Kim B-J, Ryu J, Lee J Y, Baik J M, Hong K. Electrospun ion gel nanofibers for flexible triboelectric nanogenerator: electrochemical effect on output power. Nanoscale 7: 16189-16194 (2015)

[9] Kok J F, Renno N O. Electrostatics in wind-blown sand. Phys Rev Lett 100: 014501 (2008)
[10] Houghton I M P, Aplin K L, Nicoll K A. Triboelectric charging of volcanic ash from the Grímsvötn. Phys Rev Lett 111: 118501 (2013)

[11] Zhang L, Hou J, Bi X. Triboelectric charging behavior of wood particles during pellet handling processes. J Loss Prev Process Ind 26: 1328-1334 (2013)

[12] Stöcker H, Rühl M, Heinrich A, Mehner E, Meyer D C. Generation of hard X-ray radiation using the triboelectric effect by peeling adhesive tape. $J$ Electrost 71: 905-909 (2013)

[13] Lu L, Yu L. Understanding low bandgap polymer PTB7 and optimizing polymer solar cells based on it. Adv Mater 26: 4413-4430 (2014)

[14] Zhang Y, Shao T. Effect of contact deformation on contact electrification: A first-principles calculation. J Phys Appl Phys 46: 235304 (2013)

[15] Lin S, Shao T. Bipolar charge transfer induced by water: Experimental and first-principles studies. Phys Chem Chem Phys 19: 29418-29423 (2017)

[16] Niu S, Wang Z L. Theoretical systems of triboelectric nanogenerators. Nano Energy 14: 161-192 (2015)

[17] Dhakar L. Study of effect of topography on triboelectric nanogenerator performance using patterned arrays. In Triboelectric Devices for Power Generation and Self-Powered Sensing Applications. Springer, Singapore, 2017: 39-66.

[18] Kornfeld M I. Frictional electrification. J Phys Appl Phys 9: 1183 (1976)

[19] Labadz A F, Lowell J. Contact charge density and penetration depth. J Electrost 26: 251-260 (1991)

[20] Sow M, Lacks D J, Sankaran R M. Effects of material strain on triboelectric charging: Influence of material properties. $J$ Electrost 71: 396-399 (2013)

[21] Thomas S W, Vella S J, Kaufman G K, Whitesides G M. Patterns of electrostatic charge and discharge in contact electrification. Angew Chem 120: 6756-6758 (2008)

[22] Hinchet R, Seung W, Kim S-W. Recent progress on flexible triboelectric nanogenerators for selfpowered electronics. ChemSusChem 8: 2327-2344 (2015)

[23] Kim S, Ha J, Kim J-B. Theoretical study on the dielectric effect on triboelectric nanogenerators. Integr Ferroelectr 176: 283-290 (2016)

[24] Sternovsky Z, Horányi M, Robertson S. Charging of dust particles on surfaces. J Vac Sci Technol Vac Surf Films 19: 2533-2541 (2001)

[25] Bera B. Literature review on triboelectric nanogenerator. Imp J Interdiscip Res 2: 1263-1271 (2016)

[26] Zhang Y, Shao T. Contact electrification between polymers and steel. J Electrost 71: 862-866 (2013) 
[27] Lowell J, Rose-Innes A C. Contact electrification. Adv Phys 29: 947-1023 (1980)

[28] Harper W R. The Volta effect as a cause of static electrification. Proc R Soc Lond A 205: 83-103 (1951)

[29] Williams M W. Triboelectric charging of insulating polymerssome new perspectives. AIP $A d v$ 2: 010701 (2012)

[30] Pan S, Zhang Z. Triboelectric effect: A new perspective on electron transfer process. J Appl Phys 122: 144302 (2017)

[31] Davies D K. Charge generation on dielectric surfaces. J Phys Appl Phys 2: 1533 (1969)

[32] Naik S, Mukherjee R, Chaudhuri B. Triboelectrification: A review of experimental and mechanistic modeling approaches with a special focus on pharmaceutical powders. Int J Pharm 510: 375-385 (2016)

[33] Trigwell S, Mazumder M K, Pellissier R. Tribocharging in electrostatic beneficiation of coal: Effects of surface composition on work function as measured by x-ray photoelectron spectroscopy and ultraviolet photoelectron spectroscopy in air. J Vac Sci Technol Vac Surf Films 19: 1454-1459 (2001)

[34] Pan S, Zhang Z, Yin N. Time- \& load-dependence of triboelectric effect. Sci Rep 8: 2470 (2018)

[35] Gallo C F, Lama W L. Some charge exchange phenomena explained by a classical model of the work function. $J$ Electrost 2: 145-150 (1976)

[36] Diaz A F, Felix-Navarro R M. A semi-quantitative triboelectric series for polymeric materials: The influence of chemical structure and properties. J Electrost 62: 277-290 (2004)

[37] Sickafoose A A, Colwell J E, Horányi M, Robertson S. Experimental investigations on photoelectric and triboelectric charging of dust. J Geophys Res Space Phys 106: 83438356 (2001)

[38] Son K-A, Kim H I, Houston J E. Role of stress on charge transfer through self-assembled alkanethiol monolayers on Au. Phys Rev Lett 86: 5357-5360 (2001)

[39] Akbulut M, Godfrey Alig A R, Israelachvili J. Triboelectrification between smooth metal surfaces coated with self-assembled monolayers (SAMs). J Phys Chem B 110: 22271-22278 (2006)

[40] Kron A, Reitberger T, Stenberg B. Luminescence from $\gamma$ and $\beta$-irradiated HDPE and LLDPE. Polym Int 42: 131-137 (1997)

[41] Cubero D, Quirke N, Coker D F. Electronic transport in disordered n-alkanes: From fluid methane to amorphous polyethylene. J Chem Phys 119: 2669-2679 (2003)

[42] Lacks D J, Duff N, Kumar S K. Nonequilibrium accumulation of surface species and triboelectric charging in single component particulate systems. Phys Rev Lett 100: 188305 (2008)
[43] Jean, M. S., Hudlet, S., Guthmann, C. \& Berger, J. Local triboelectricity on oxide surfaces. Eur. Phys. J. B - Condens. Matter Complex Syst. 12, 471-477 (1999).

[44] Castle G S P. Contact charging between insulators. J Electrost 40-41: 13-20 (1997)

[45] Liu C, Bard A J. Electrostatic electrochemistry at insulators. Nat Mater 7: 505 (2008)

[46] Liu C, Bard A J. Electrons on dielectrics and contact electrification. Chem Phys Lett 480: 145-156 (2009)

[47] Mizes H A, Conwell E M, Salamida D P. Direct observation of ion transfer in contact charging between a metal and a polymer. Appl Phys Lett 56: 1597-1599 (1990)

[48] Alexander A J. Interfacial ion-transfer mechanism for the intense luminescence observed when opening self-seal envelopes. Langmuir 28: 13294-13299 (2012)

[49] Gooding D M, Kaufman G K. Tribocharging and the triboelectric series. In Encyclopedia of Inorganic and Bioinorganic Chemistry. John Wiley \& Sons, Ltd, 2011.

[50] Huheey H, James E. Inorganic Chemistry: Principles of Structure and Reactivity. Pearson Education India.

[51] Williams M W. Triboelectric charging of insulators - mass transfer versus electrons/ions. J Electrost 70: 233-234 (2012)

[52] Salaneck W R, Paton A, Clark D T. Double mass transfer during polymer-polymer contacts. J Appl Phys 47: 144-147 (1976)

[53] Wang A, Gil D, Holonga M, Lacks D J, Baytekin H T, Sankaran R M, Yavuz Z. Dependence of triboelectric charging behavior on material microstructure. Phys Rev Mater 1: 035605 (2017)

[54] Chung Y G, Lacks D J. Atomic mobility in strained glassy polymers: The role of fold catastrophes on the potential energy surface. J Polym Sci Part B Polym Phys 50: 1733-1739 (2012)

[55] Jacobs T D B, Carpick R W. Nanoscale wear as a stressassisted chemical reaction. Nat Nanotechnol 8: 108 (2013)

[56] Jacobs T D B, Gotsmann B, Lantz M A, Carpick R W. On the application of transition state theory to atomic-scale wear. Tribol Lett 39: 257-271 (2010)

[57] Hänggi P, Talkner P, Borkovec M. Reaction-rate theory: Fifty years after Kramers. Rev Mod Phys 62: 251-341 (1990)

[58] Su Y, Chen J, Wu Z, Jiang Y. Low temperature dependence of triboelectric effect for energy harvesting and self-powered active sensing. Appl Phys Lett 106: 013114 (2015)

[59] Apodaca M M, Wesson P J, Bishop K J M, Ratner M A, Grzybowski B A. Contact electrification between identical materials. Angew Chem 122: 958-961 (2010) 
[60] Lowell J, Truscott W S. Triboelectrification of identical insulators. II. Theory and further experiments. J Phys Appl Phys 19: 1281 (1986)

[61] Shinbrot T, Komatsu T S, Zhao Q. Spontaneous tribocharging of similar materials. EPL Europhys Lett 83: 24004 (2008)

[62] Baytekin H T, Patashinski A Z, Branicki M, Baytekin B, Soh S, Grzybowski B A. The mosaic of surface charge in contact electrification. Science 333: 308-312 (2011)

[63] Pham R, Virnelson R C, Sankaran R M, Lacks D J. Contact charging between surfaces of identical insulating materials in asymmetric geometries. J Electrost 69: 456-460 (2011)

[64] Forward K M, Lacks D J, Sankaran R M. Particle-size dependent bipolar charging of Martian regolith simulant. Geophys Res Lett 36: L13201 (2009)

[65] Miura T, Koyaguchi T, Tanaka Y. Measurements of electric charge distribution in volcanic plumes at Sakurajima Volcano, Japan. Bull Volcanol 64: 75-93 (2002)

[66] Inculet I I, Peter Castle G S, Aartsen G. Generation of bipolar electric fields during industrial handling of powders. Chem Eng Sci 61: 2249-2253 (2006)

[67] Melnik O, Parrot M. Electrostatic discharge in Martian dust storms. J Geophys Res Space Phys 103: 29107-29117 (1998)

[68] Farrell W M, Delory G T, Cummer S A, Marshall J R. A simple electrodynamic model of a dust devil. Geophys Res Lett 30: 2050 (2003)

[69] Rowley G. Quantifying electrostatic interactions in pharmaceutical solid systems. Int J Pharm 227: 47-55 (2001)

[70] Forward K M, Lacks D J, Sankaran R M. Charge segregation depends on particle size in triboelectrically charged granular materials. Phys Rev Lett 102: 028001 (2009)

[71] Forward K M, Lacks D J, Sankaran R M. Triboelectric charging of lunar regolith simulant. J Geophys Res Space Phys 114: A10109 (2009)

[72] Kaalund C J, Haneman D. Positive ion and electron emission from cleaved Si and Ge. Phys Rev Lett 80: 3642-3645 (1998)

[73] Sakaguchi M, Shimada S, Kashiwabara H. Mechanoions produced by mechanical fracture of solid polymer. 6. A generation mechanism of triboelectricity due to the reaction of mechanoradicals with mechanoanions on the friction surface. Macromolecules 23: 5038-5040 (1990)

[74] Zimmerman K A, Langford S C, Dickinson J T, Dion R P.
Electron and photon emission accompanying deformation and fracture of polycarbonate. J Polym Sci Part B Polym Phys 31: 1229-1243 (1993)

[75] Yang W, Chen J, Zhu G, Wen X, Bai P, Su Y, Lin Y, Wang Z. Harvesting vibration energy by a triple-cantilever based triboelectric nanogenerator. Nano Res 6: 880-886 (2013)

[76] Wang Z L, Song J. Piezoelectric nanogenerators based on zinc oxide nanowire arrays. Science 312: 242-246 (2006)

[77] Su L, Li ,H Y, Wang Y, Kuang S Y, Wang Z L, Zhu G. Coupling of photoelectric and triboelectric effects as an effective approach for PZT-based high-performance selfpowered ultraviolet photodetector. Nano Energy 31: 264-269 (2017)

[78] Park J, Yun K S. Hybrid energy harvester based on piezoelectric and triboelectric effects. In 2016 IEEE 29th International Conference on Micro Electro Mechanical Systems (MEMS), 2016: 41-42.

[79] Rescaglio A, Schockmel J, Vandewalle N, Lumay G. Combined effect of moisture and electrostatic charges on powder flow. EPJ Web Conf 140: 13009 (2017)

[80] Clint J H, Dunstan T S. Acid-base components of solid surfaces and the triboelectric series. EPL Europhys Lett 54: 320 (2001)

[81] Wiles J A, Fialkowski M, Radowski M R, Whitesides G M, Grzybowski B A. Effects of surface modification and moisture on the rates of charge transfer between metals and organic materials. J Phys Chem B 108: 20296-20302 (2004)

[82] Ducati T R D, Simões L H, Galembeck F. Charge partitioning at gas-solid interfaces: Humidity causes electricity buildup on metals. Langmuir 26: 13763-13766 (2010)

[83] Gouveia R F, Galembeck F. Electrostatic charging of hydrophilic particles due to water adsorption. J Am Chem Soc 131: 11381-11386 (2009)

[84] Adamson A. Physical Chemistry of Surfaces. Wiley, India, 1990.

[85] Horn R G, Smith D T, Grabbe A. Contact electrification induced by monolayer modification of a surface and relation to acid-base interactions. Nature 366: 442 (1993)

[86] Friedle S, Thomas S W. Controlling contact electrification with photochromic polymers. Angew Chem Int Ed 49: 7968-7971 (2010) 


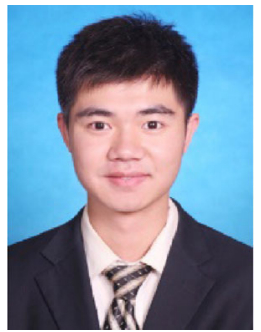

Shuaihang PAN. He received his B.S. degree in Mech. Eng. and B.A. degree in German in 2016 from Shanghai Jiao Tong University, Shanghai, China. He is now working as a graduate researcher at University

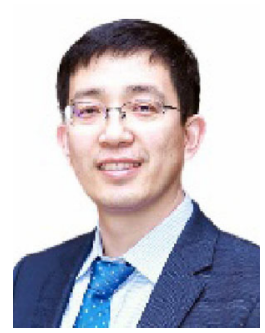

Zhinan ZHANG. He received his Ph.D. degree in 2011 from Shanghai Jiao Tong University, Shanghai, China. After that he was a post doctor in Shanghai Jiao Tong University. He is now working as of California-Los Angeles (UCLA) in pursuit of a Ph.D. degree. His research focuses on triboelectric effect, novel nanocomposites, and electrical/thermal transport phenomena.

an associate professor in the School of Mechanical Engineering, Shanghai Jiao Tong University. His research interests include computational design and analysis of tribosystems, and theory and methods of design engineering and innovation. 